\title{
False rifampicin resistant results using Xpert MTB/RIF on urine samples in hospitalised HIV-infected patients
}

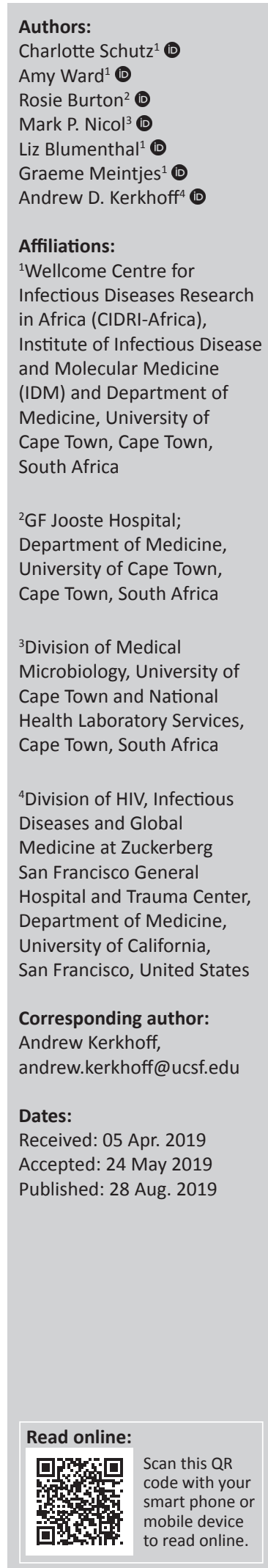

Background: A small proportion of false rifampicin resistant results have previously been reported using GeneXpert MTB/RIF version G4 on sputum samples; however, this has not been investigated for urine samples in HIV-associated tuberculosis (TB).

Objectives: We sought to determine the proportion of false rifampicin resistant results using Xpert MTB/RIF version G4 on urine samples among HIV-infected inpatients investigated for TB.

Methods: Hospitalised HIV-infected patients undergoing systematic TB testing from two cohorts in Cape Town, South Africa, were enrolled. All patients with $\geq 1$ urine Xpert result available were included. Rifampicin resistant urine Xpert results were classified into three mutually exclusive groups: (1) true rifampicin resistance, (2) false rifampicin resistance or (3) unknown after review of available microbiologic and clinical data.

Results: Overall, 1171 patients were included, from whom a total of 1704 urine Xpert results were available on unconcentrated and/or concentrated urine samples. There were 416 samples positive for TB (24.4\% [95\% CI 22.4-26.5]), of which 43/413 (10.4\% [95\% CI 7.6-13.8]) were rifampicin resistant (after excluding three results that were falsely positive due to contamination). Of 43 rifampicin resistant Xpert results (among 40 patients), 30 were classified as true resistance, 11 as false resistance and 2 could not be classified. Excluding unclassifiable results, 30/41 results were confirmed as true-positive urine Xpert rifampicin resistance (positive predictive value: $73.2 \%$ [95\% CI 57.1-85.8]).

Conclusion: Urine Xpert testing showed a high proportion of false rifampicin resistance results. Urine Xpert rifampicin resistant results should be interpreted cautiously and confirmed when possible.

Keywords: HIV; AIDS; Tuberculosis; Xpert; Rifampicin resistance; False resistance.

\section{Introduction}

Tuberculosis (TB) remains the leading cause of death in people living with HIV, contributing to one-in-three AIDS-related deaths. ${ }^{1}$ Timely diagnosis of $\mathrm{TB}$ in such patients remains challenging because of non-specific presentations and disseminated disease. ${ }^{2,3,4}$ Gene Xpert MTB/RIF, an automated nucleic acid amplification test, is capable of providing results in a few hours and represents an important breakthrough for diagnosing HIV-associated TB. Importantly, Xpert also rapidly detects rifampicin resistance, without need for an additional sample or cartridges. It has been endorsed by the World Health Organization (WHO) since 2010. Sputum Xpert (or Xpert Ultra where available) is currently recommended by the WHO as the initial diagnostic test in patients with suspected HIV-associated TB or multi-drug resistant (MDR) TB. ${ }^{5}$ In those with microbiologically confirmed TB, Xpert MTB/RIF is also recommended by the $\mathrm{WHO}$ as a first-line assay for the rapid detection of rifampicin-resistance. It is therefore an important tool in tackling the growing global health challenge of drug resistant (DR)-TB. However, the WHO does not currently have a recommendation regarding the use of Xpert MTB/RIF in urine owing to an insufficient amount of data on the performance and utility of this assay in urine specimens. ${ }^{6}$

In concordance with WHO guidelines, sputum Xpert was implemented as the initial diagnostic evaluation in those with suspected TB and DR-TB in South Africa as well as other countries ${ }^{7}$ and in South Africa, it has now been replaced with the updated Xpert Ultra cartridge. Although

How to cite this article: Schutz C, Ward A, Burton R, et al. False rifampicin resistant results using Xpert MTB/RIF on urine samples in hospitalised HIV-infected patients. S Afr J HIV Med. 2019;20(1), a978. https://doi.org/10.4102/sajhivmed.v20i1.978

Copyright: ( 2019. The Authors. Licensee: AOSIS. This work is licensed under the Creative Commons Attribution License. 
Xpert has not been associated with a mortality reduction in most trials to date ${ }^{8,9,10}$ its implementation has been associated with overall shorter times to starting anti-TB therapy, including DR TB. ${ }^{8,11,12,13}$ It has also increased the diagnostic yield by $1.4 \%-15 \%$ (compared to sputum microscopy) in clinical trials in Sub-Saharan Africa, Brazil and Indonesia. $8,9,10,11,13,14,15,16$

Against the backdrop of improved case detection, previous studies have reported on false rifampicin resistance results associated with the Xpert MTB/RIF assay, and meta-analyses found the overall specificity of the Xpert for rifampicinresistance in sputum samples to be $98 \%$ (i.e. $2 \%$ showed false rifampicin resistant results) and $99 \%$ in extra-pulmonary samples. ${ }^{17,18}$ This however appeared to be associated in part with earlier Xpert cartridge generations. ${ }^{19}$ An implementation study from South Africa found the Xpert G4 cartridge to have excellent positive predictive value for rifampicin resistance of $99.5 \%$ (95\% CI 98.5-100) in sputum samples. ${ }^{20}$

We have previously found that among HIV-patients requiring acute medical hospitalisation, testing of a single concentrated urine sample detected 2.2 times more TB cases than sputum Xpert testing, largely because of the inability of sick inpatients to produce a sputum sample. ${ }^{21}$ Additionally, a recent randomised, multi-country trial found that the addition of rapid urine-based assays (including urine Xpert) to sputum Xpert testing was associated with reduced mortality among hospitalised HIVinfected patients in sub-group analyses. ${ }^{22}$ This suggests that urine-based testing using Xpert may have an important role in the TB diagnostic algorithm among hospitalised patients with advanced HIV, especially those too ill to produce a sputum sample. However, the proportion of false-positive rifampicin resistance results using Xpert on urine samples has not been reported. We sought to determine the proportion of urine Xpert false rifampicin resistance results among hospitalised HIV-infected patients being investigated for HIV-associated TB in Cape Town, South Africa.

\section{Methods}

\section{Patients and setting}

Patients from two parent cohort studies were included. In the first, patients were recruited at GF Jooste Hospital, South Africa from June 2012 to October 2013. Unselected HIVinfected patients admitted to the medical wards were recruited within $24 \mathrm{~h}$ of admission, regardless of TB treatment at the time of admission. ${ }^{21,23}$ GF Jooste Hospital was closed at the end of 2013 and two new hospitals (including Khayelitsha Hospital) were opened serving the same communities at the time the second study was conducted. The second study was undertaken at Khayelitsha Hospital from January 2014 until October 2016 and recruited HIV-infected patients with a low CD4 T-cell count $(<350$ cells $/ \mu \mathrm{L})$ admitted to hospital with a suspected new diagnosis of TB. Patients already on TB treatment were excluded from this study. Sputum, blood and urine samples were systematically obtained (when possible) as part of both study protocols and submitted for mycobacteriology (TB culture and/or Xpert). Information about any additional specimens, that were clinically indicated and collected by the medical teams were also recorded - for example, lymph node aspirates, cerebrospinal fluid TB cultures, pleural TB cultures and urine TB cultures.

In the first study, two cases of false urine rifampicin resistance occurred 3 months after study initiation (Appendix Table 1-A1 - patients JTBS097 \& JTBS099). Both patients' urine Xpert samples were collected after a sample was taken from an MDR patient earlier on the same day. It was determined that both samples were likely contaminated due to inadequate cleaning of the reusable bedpan, although laboratory cross-contamination could not be ruled out. We subsequently introduced single-use disposable bed pans (Litha Healthcare Group, Johannesburg, South Africa) and these were used for the remainder of the Jooste Hospital TB study and the duration of the Khayelitsha Hospital TB study. There were no repeat episodes of suspected cross-contamination. Urine was transferred to a polypropylene tube using a sterile syringe. Patients from both cohort studies had urine Xpert testing performed. Demographic details and clinical symptoms were recorded for all patients at study entry. Patients were managed by the hospital and clinic staff, and all TB diagnostic test results were made available by study staff and could be utilised to inform patient care.

\section{Laboratory methods}

Urine Xpert testing for both studies was performed at the Groote Schuur Hospital National Health Laboratory Service laboratory using Xpert MTB/RIF Assay G4 version 5. All specimens were processed using standardised protocols and quality assurance procedures as previously described. ${ }^{24}$ In brief, for the GF Jooste Hospital study, Xpert testing of urine samples was conducted in two ways on each sample. The first method (unconcentrated) utilised $2.0 \mathrm{~mL}$ of fresh urine that was centrifuged, resuspended in $0.75 \mathrm{~mL}$ phosphate buffer and then tested using Xpert. ${ }^{25}$ The second method (concentrated) used a $30 \mathrm{~mL}-40 \mathrm{~mL}$ urine sample that was centrifuged at $3000 \mathrm{~g}$ for $15 \mathrm{~min}$. The resultant supernatant was removed and the pellet was resuspended in the residual urine volume (without the addition a phosphate buffer); $0.75 \mathrm{~mL}$ was then tested using Xpert. ${ }^{21}$ For both methods, Xpert sample reagent $(1.5 \mathrm{~mL})$ was added to the samples as per manufacturer's instructions. The Khayelitsha Hospital study only used Xpert testing on concentrated urine samples and was undertaken using the same methods as described above. The reference standard for drug resistance, including rifampicin resistance for both studies, was a molecular line probe assay (MTBDR plus; Hain Lifescience Nehren, Germany) undertaken on culture isolates from any clinical specimen (not necessarily urine).

\section{Analysis}

Patient populations were from overlapping referral areas in the Cape Town townships and both cohorts included 
HIV-infected patients requiring medical admission and had detailed TB investigations performed. Urine Xpert rifampicin resistance results were classified by two authors independently by first assessing all available microbiological results (including culture, Xpert and line probe assay) on all clinical samples. In cases where it was not possible to classify urine Xpert rifampicin resistance results by assessing microbiological results from other clinical samples, the type of TB treatment, response to treatment and vital status at 12 weeks were also considered. All patients with urine Xpert rifampicin resistant results were assigned to one of the three mutually exclusive groups: (1) true rifampicin resistant urine Xpert (patients who had rifampicin-resistant TB confirmed by culture or Xpert on other clinical samples) (2) false rifampicin resistant urine Xpert (patients who did not have rifampicin-resistant $\mathrm{TB}$ present on additional clinical samples and had a clinical course that was not compatible with drug-resistant TB), (3) unknown (insufficient microbiological and clinical evidence to classify a patient's urine Xpert rifampicin resistant result). Furthermore, patients with true urine Xpert rifampicin resistance were classified as having heteroresistance if additional independent sample/s from the same clinical episode demonstrated both a rifampicin-susceptible and a rifampicinresistant Mycobacterium tuberculosis (MTB) isolate, i.e. discordant results from two different clinical specimens in the same patient. Two patients (contributing three urine Xpert rifampicin resistance results) were determined to have false urine Xpert rifampicin resistance; this occurred within 3 months of initiating the first study, and was prior to the introduction of single use disposable bedpans (see details above). This led to the introduction of single-use disposable bedpans and avoided further such cases.

\section{Ethical consideration}

Approval for both studies was obtained from the University of Cape Town Faculty of Health Sciences Human Research Ethics Committee and patients provided written informed consent according to the approved study protocols.

\section{Results}

There were 585 patients from the GF Jooste Hospital cohort and 586 patients from the Khayelitsha Hospital cohort with urine Xpert results available for a total of 1171 hospitalised HIV-infected patients. Overall 1704 urine Xpert results were available from 1171 patients, of which 554 were performed on unprocessed urine samples and 1150 on concentrated urine samples (Figure 1). Baseline characteristics of the two cohorts were similar. (Table 1).

Among 1704 urine Xpert results, there were $416(24.4 \%$ [95\% CI 22.4-26.5]) samples that tested positive for MTB and 46 results indicating rifampicin resistance among 42 patients (Figure 1). After excluding three results (from two patients) that were determined to be caused by contamination, 43 results from 40 patients remained ( $n=43$ /413; prevalence $10.4 \%$ [95\% CI 7.6-13.8]) and were further classified.
The majority of rifampicin resistance results $(n=30 / 43 ; 69.8 \%$ [95\% CI 53.9-82.8]) were classified as true urine Xpert rifampicin resistance based on the results from other independent clinical samples. Eleven $(11 / 43,25.6 \%$ [95\% CI 13.5-41.2]) results were classified as false rifampicin resistance and two further results (one from each study) could not be classified. Thus, by the most conservative estimate (excluding 2 unknown results), $n=30 / 41$ results were confirmed as true urine Xpert rifampicin resistant results, for a positive predictive value of $73.2 \%$ (95\% CI 57.185.8). Comprehensive details for each patient with urine Xpert rifampicin resistance were reported in Appendix Table 1-A1.

TABLE 1: Baseline characteristics of Jooste Hospital tuberculosis study and Khayelitsha Hospital tuberculosis study patients.

\begin{tabular}{|c|c|c|c|c|}
\hline \multirow[t]{2}{*}{ Variable } & \multicolumn{2}{|c|}{$\begin{array}{c}\text { Jooste Hospital study } \\
(n=585) \\
\end{array}$} & \multicolumn{2}{|c|}{$\begin{array}{l}\text { Khayelitsha Hospital study } \\
\qquad(n=586)\end{array}$} \\
\hline & $n$ & $\%$ or IQR & $n$ & $\%$ or IQR \\
\hline \multicolumn{5}{|l|}{ Sex } \\
\hline Female & 338 & 57.8 & 307 & 52.4 \\
\hline Male & 247 & 42.2 & 279 & 47.6 \\
\hline Age, years & 35.3 & $28.9,41.4$ & 35.9 & $30.8,43.9$ \\
\hline \multicolumn{5}{|l|}{ ART status } \\
\hline Defaulted ART & 113 & 19.3 & 140 & 24.1 \\
\hline ART naive & 209 & 35.7 & 222 & 38.3 \\
\hline Currently on ART & 263 & 45.0 & 218 & 37.6 \\
\hline \multicolumn{5}{|l|}{ TB history } \\
\hline Previous TB & 263 & 45.1 & 268 & 45.7 \\
\hline Unknown TB history & 2 & 0.3 & 23 & 3.9 \\
\hline CD4, cells/mL & 134 & 53,275 & 66 & 24,138 \\
\hline HIV viral load, log copies/mL & 4.2 & $1.6,5.5$ & 5.2 & $3.8,5.7$ \\
\hline $\begin{array}{l}\text { Established on TB } \\
\text { treatment at enrolment }\end{array}$ & 158 & 27 & - & - \\
\hline
\end{tabular}

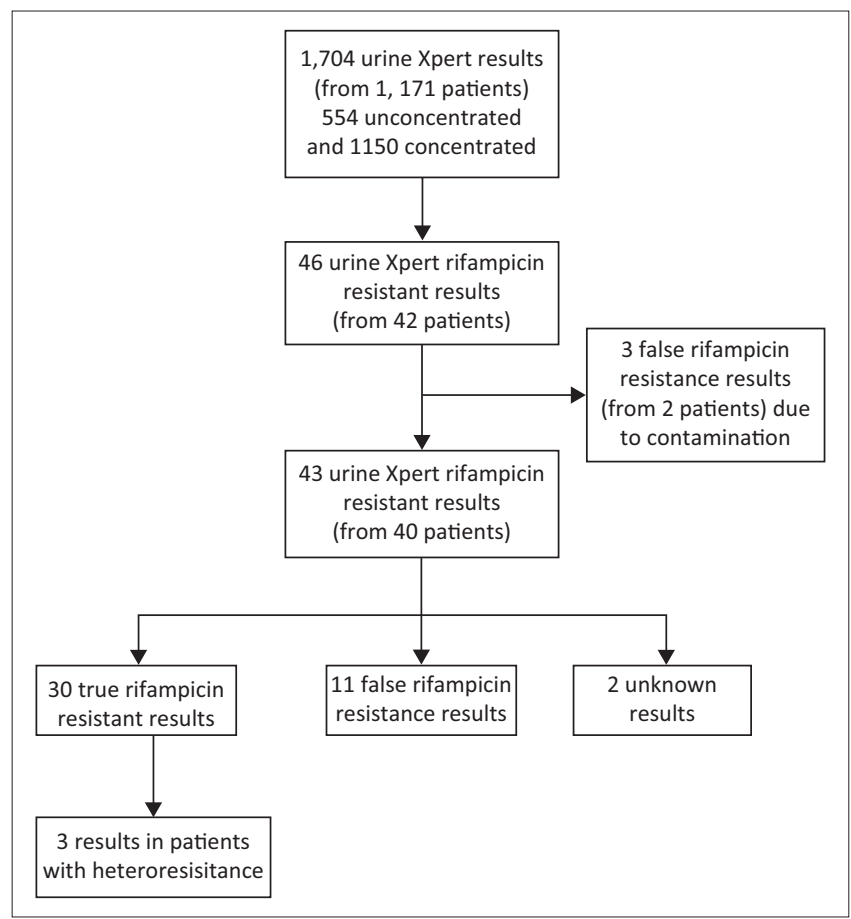

FIGURE 1: Overview of urine Xpert rifampicin resistance results from two cohorts of hospitalised HIV-patients in Cape Town, South Africa. 
False urine rifampicin resistance results were more commonly observed in the Jooste Hospital study: 9/18 (50\%) results compared with $2 / 25(8 \%)$ in the Khayelitsha Hospital study (Figure 2). The Jooste Hospital study enrolled not only patients not yet on TB treatment but also those already established on TB treatment, whereas the Khayelitsha Hospital study excluded patients who were already on TB treatment at the time of admission. In the Jooste Hospital study, there were $n=14$ results (one unknown rifampicin resistant result) from patients on $\mathrm{TB}$ treatment at enrolment and $n=4$ results from patients not on TB treatment at enrolment and among these, $n=7 / 13$ (53.8\%) and $n=2 / 4(50 \%)$ had false rifampicin resistant urine Xpert results, respectively. Therefore, in both cohorts and excluding two results that could not be classified, among patients not on TB therapy, $n=24 / 28$ (85.7\% [95\% CI 67.3-96.0] had true positive urine Xpert resistance results compared to $n=7 / 13$ (53.8\% [95\% CI 25.1-80.8] among those receiving $\mathrm{TB}$ therapy at study enrolment. This suggests that the positive predictive value of Xpert MTB/ RIF for rifampicin resistance is higher among those not on TB treatment compared with those who were already established on TB treatment.

Twelve-week mortality for patients with urine Xpert rifampicin resistant results was 30\% $(n=12 / 40)$ and $7.5 \%$ ( $n=3 / 40)$ were lost to follow-up. No deaths were observed among the 10 patients (accounting for 11 results) with false urine Xpert rifampicin resistance. Limited details regarding

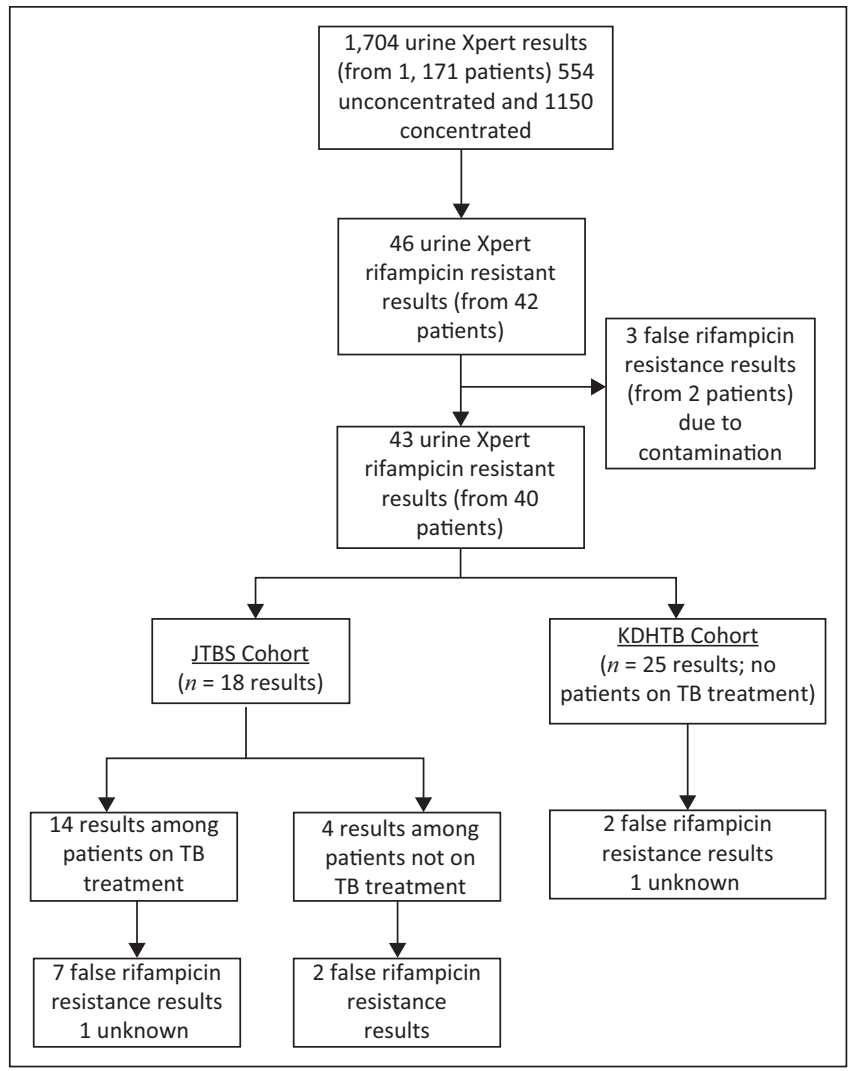

FIGURE 2: Urine Xpert rifampicin resistance results separated by cohort and tuberculosis treatment status.
Xpert probe features for the two patients with false rifampicin resistance in the Khayelitsha Hospital study were available. The clinical microbiologists' comment for patient KDHTB479 indicated that there was a very low load with a double mutation detected by a delay in probes $\mathrm{D}$ and $\mathrm{E}$ and that the result was likely false positive. In patient KDHTB439 there was a failure of probe $\mathrm{D}$ to bind in the isolate and a repeat sample was requested that demonstrated RIF susceptibility. We were unable to obtain information about the probe features for samples of the JTBS study.

Three patients ( $n=3 / 40,7.5 \%)$ with a confirmed rifampicin resistant urine Xpert result had evidence of likely heteroresistant infection. The first patient (Appendix Table 1-A1 - KDHTB203) cultured a drug susceptible isolate from blood (MycoF/lytic bottle), sputum and urine samples but also a rifampicin resistant isolate from sputum during the same admission. The second patient (KDHTB531) cultured a drug-sensitive isolate from blood as well as a drug-resistant isolate from sputum during the same admission. The third patient (JTBS463) was originally started on drug-sensitive TB treatment after a prior sputum Xpert and abscess aspirate culture both showed rifampicin susceptible isolates. One month after starting TB treatment, the patient was admitted for TB immune reconstitution inflammatory syndrome (IRIS). Shortly after discharge, the patient was readmitted for gastroenteritis and was clinically deteriorating despite drug-sensitive TB treatment. At this time, two urine Xpert results showed rifampicin resistance; however, the patient died shortly after receipt of urine Xpert results.

\section{Discussion}

In this study, which included hospitalised HIV-infected patients systematically investigated for TB, the overall proportion of urine Xpert rifampicin resistance results was $10.4 \%(n=43 / 413)$; however, the positive predictive value of urine Xpert MTB/RIF for rifampicin resistance was only $73.2 \%(n=30 / 41)$.

The correct identification of drug-resistant TB has important implications for both the individuals' health as well as for public health. For the patient, a false rifampicin resistance result may result in not only over-treatment with more toxic drugs that are less efficacious for drugsensitive TB, but also significantly and unnecessarily prolong treatment times. In high burden, under-resourced settings, a false rifampicin resistance may have important resource implications by resulting in additional drug susceptibility testing, significantly more expensive treatment costs and unnecessary community contact tracing. ${ }^{26}$ Thus, any test that detects DR TB should ideally have very high specificity. Under the best-case scenario when results were restricted to those not receiving TB treatment, we found that Xpert testing of rifampicin resistance on urine samples did not achieve sufficiently high positive predictive value $(86 \%)$ to be the sole/ 
definitive test for drug-resistant TB identification. This, however, needs to be evaluated in additional settings.

Xpert Ultra is an updated, next-generation sample cartridge for the Xpert platform that is now recommended by the $\mathrm{WHO}$ as a replacement for the current Xpert MTB/RIF cartridge $\mathrm{e}^{27,28}$ and has been implemented in South Africa. It provides increased sensitivity for the detection of MTB in sputum (especially smear-negative and pauci-baciliary disease). Xpert Ultra utilises a new melt curve analysis to detect RIF-resistance; however, its diagnostic accuracy (including specificity) for the detection of rifampicin resistance is similar to that of Xpert. ${ }^{29}$ The results of this study suggest that urine Xpert Ultra rifampicin resistance results should be interpreted cautiously and confirmed by alternative drug susceptibility testing (either phenotypic or alternative genotypic assays) until the specificity of Xpert Ultra for rifampicin resistance detection has been confirmed to be adequately high to warrant stand-alone testing on urine samples.

Of interest, in this cohort we describe three patients with a confirmed urine Xpert rifampicin resistance result who also had drug-sensitive strains from independent samples during the same admission suggesting likely heteroresistance (either polyclonal infection or acquired heteroresistance). The prevalence of heteroresistance in MTB infections has previously been described..$^{30,31,32}$ Although not well-studied, these are likely associated with increased rates of treatment failure for the individual ${ }^{31}$ and could complicate TB control efforts at a population level. Xpert may miss heteroresistance if used as a standalone test for the detection of rifampicin resistance, however, early studies show that Xpert Ultra may detect heteroresistance when the resistant DNA comprises $5 \%$ or more of the sample. ${ }^{28}$

Strengths of this study include a large number of urine Xpert rifampicin results from two geographically and clinically comparable cohorts where patients were prospectively recruited and underwent systematic testing for TB. Additionally, all TB assays including urine Xpert testing were performed at the same laboratory according to standard protocols. After an error yielded two likely false Xpert rifampicin resistant urine cases due to contamination soon after recruitment initiation, disposable bedpans (single-use) were implemented for the duration of both studies. We therefore recommend that clinicians use single-use specimen collection bedpans and containers when utilising Xpert or Xpert Ultra testing on urine to prevent DNA-crosscontamination between samples.

The reason(s) for the high proportion of false positive urine rifampicin resistance is not entirely clear, but the proportion was higher among those already receiving TB therapy. A limitation of this study is that we did not have data available to systematically evaluate the Xpert probe features associated with our classification of false rifampicin resistance.
Different methods of drug susceptibility testing could explain discrepant results in some cases. ${ }^{33,34}$ The majority of drug susceptibility testing on cultured isolates in both studies was PCR-based; however, we also captured results of all TB tests performed in-service and cannot reliably differentiate between drug susceptibility testing performed with other methods such as liquid or solid media for all samples for the duration of the study. Because of the early implementation of disposable bedpans, we do not suspect undetected contamination beyond that described above. Furthermore, because most patients with positive urine rifampicin results did not have paired urine culture isolates available for further genotypic or phenotypic drug-susceptibility testing, patients classified as having false positive rifampicin results may have had heteroresistance with compartmentalised true rifampicin-resistant urinary $\mathrm{TB}$ and rifampicin-susceptible TB at other anatomic sites. However, the favourable clinical course of most of these patients on first-line drug-sensitive TB treatment counts against this possibility. Notably, a large proportion of false positive rifampicin results were among those already receiving anti-TB therapy, where $50 \%$ of urine Xpert rifampicin resistance results were classified as false resistance; this suggests that further caution should be applied when interpreting urine Xpert rifampicin resistance results in treatment-experienced patients.

An additional limitation of the study is that sequencing of isolates was not performed as part of either study. Sequencing of the rpoB gene would have been particularly useful in the cases that we could not classify as true or false resistance and the heteroresistant cases. Furthermore, urine TB cultures were not routinely performed in either study and it may have been useful to compare drug susceptibility results on isolates cultured from urine samples collected at the same time as the urine Xpert samples.

In conclusion, urine testing using Xpert provides important diagnostic yield for hospitalised HIV-infected patients being investigated for HIV-associated TB, especially in those unable to produce sputum samples. Although the overall proportion of patients with urine Xpert rifampicin resistance in this cohort was relatively low, the proportion of those classified as false rifampicin resistance was substantially higher than has previously been reported on sputum. Urine Xpert rifampicin resistant results should therefore be interpreted with caution, repeated on a second sample in patients at lowrisk for drug resistant TB (as currently recommended by the WHO for sputum samples) and confirmed using additional culture-based or molecular assays when possible. Whether these findings apply to Xpert Ultra is an issue that requires further study.

\section{Acknowledgements}

The late Stephen D. Lawn was PI on the Jooste Hospital study. The investigators are grateful to the clinical and administrative staff of the Western Cape Department of Health. 


\section{Competing interests}

The authors have no conflict of interests.

\section{Authors' contributions}

G.M. R.B., C.S., L.B. A.W. and G.M. were responsible for patient recruitment and overseeing the individual study sites. A.D.K. and C.S. were responsible for the database. C.S. and A.D.K. designed and performed the analyses with input from G.M. and M.P.N. was responsible for the mycobacteriology. C.S. and A.D.K. wrote the first draft of the article and G.M. gave input to further drafts. All other authors commented on a draft and approved the final version of the article.

\section{Funding Information}

G.M. was supported by the Wellcome Trust (098316), the South African Research Chairs Initiative of the Department of Science and Technology and National Research Foundation (NRF) of South Africa (Grant No 64787), NRF incentive funding (UID: 85858) and the South African Medical Research Council through its TB and HIV Collaborating Centres Programme with funds received from the National Department of Health (RFA\# SAMRCRFA-CC: TB/HIV/AIDS-01-2014). C.S. is funded by the South African Medical Research Council under the National Health Scholars Programme. A.D.K. was supported by the National Institute of Allergy and Infectious Diseases (Grant No T32 AI060530). The funders had no role in the study design, data collection, data analysis, data interpretation or writing of this report. The opinions, findings and conclusions expressed in this manuscript reflect those of the authors alone.

\section{Data availability statement}

The data sets generated during and/or analysed during the current study are available from the corresponding author on reasonable request.

\section{Disclaimer}

The views expressed in the article are those of the authors and not an official position of the institution or funder.

\section{References}

1. Global Tuberculosis Report 2016. Geneva: World Health Organization; 2016.

2. Kyeyune $R$, Den Boon $S$, Cattamanchi $A$, et al. Causes of early mortality in HIV-infected TB suspects in an East African referral hospital. J Acquir Immune Defic Syndr. 2010;55(4):446-450. https://doi.org/10.1097/QAl.0b013e3181eb611a 3. Macpherson $P$, Dimairo $M$, Bandason T, et al. Risk factors for mortality in smear-
negative tuberculosis suspects: A cohort study in Harare, Zimbabwe. Int I Tuberc Lung Dis. 2011;15(10):1390-1396. https://doi.org/10.5588/ijtld.11.0056

4. Crump JA, Ramadhani HO, Morrissey $A B$, et al. Bacteremic disseminated tuberculosis in sub-saharan Africa: A prospective cohort study. Clin Infect Dis. 2012;55(2):242-250. https://doi.org/10.1093/cid/cis409

5. Organization WH. Consolidated guidelines on the use of antiretroviral drugs for treating and preventing HIV infection. Geneva: World Health Organization 2016.

6. Xpert MTB/RIF implementation manual: Technical and operational 'how-to'; practical considerations. Geneva: World Health Organization; 2014.
7. Albert $\mathrm{H}$, Nathavitharana RR, Isaacs $\mathrm{C}$, Pai $\mathrm{M}$, Denkinger $\mathrm{CM}$, Boehme CC. Development, roll-out and impact of Xpert MTB/RIF for tuberculosis: What lessons have we learnt and how can we do better? Eur Respir J. 2016;48(2): 516-525. https://doi.org/10.1183/13993003.00543-2016

8. Theron G, Zijenah L, Chanda D, et al. Feasibility, accuracy, and clinical effect of pointof-care Xpert MTB/RIF testing for tuberculosis in primary-care settings in Africa: A multicentre, randomised, controlled trial. Lancet. 2014;383(9915):424-435. https://doi.org/10.1016/S0140-6736(13)62073-5

9. Churchyard GJ, Stevens WS, Mametja LD, et al. Xpert MTB/RIF versus sputum microscopy as the initial diagnostic test for tuberculosis: A cluster-randomised trial embedded in South African roll-out of Xpert MTB/RIF. Lancet Glob Health. 2015;3(8):e450-e457. https://doi.org/10.1016/S2214-109X(15)00100-X

10. Durovni B, Saraceni V, Van den Hof S, et al. Impact of replacing smear microscopy with Xpert MTB/RIF for diagnosing tuberculosis in Brazil: A stepped-wedge clusterrandomized trial. PLoS Med. 2014:11(12):e1001766. https://doi.org/10.1371/ journal.pmed.1001766

11. Van Kampen SC, Susanto NH, Simon S, et al. Effects of introducing Xpert MTB/RIF on diagnosis and treatment of drug-resistant tuberculosis patients in Indonesia: A pre-post intervention study. PLoS One. 2015;10(6):e0123536. https://doi. org/10.1371/journal.pone.0123536

12. Iruedo J, O'Mahony D, Mabunda S, Wright G, Cawe B. The effect of the Xpert MTB/ RIF test on the time to MDR-TB treatment initiation in a rural setting: A cohort study in South Africa's Eastern Cape Province. BMC Infect Dis. 2017;17(1):91. https://doi.org/10.1186/s12879-017-2200-8

13. Cox HS, Mbhele S, Mohess N, et al. Impact of Xpert MTB/RIF for TB diagnosis in a primary care clinic with high TB and HIV prevalence in South Africa: A pragmatic randomised trial. PLoS Med. 2014;11(11):e1001760. https://doi.org/10.1371/ journal.pmed.1001760

14. Mupfumi L, Makamure B, Chirehwa M, et al. Impact of Xpert MTB/RIF on antiretroviral therapy-associated tuberculosis and mortality: A pragmatic randomized controlled trial. Open Forum Infect Dis. 2014;1(1):ofu038. https:// doi.org/10.1093/ofid/ofu038

15. Yoon C, Cattamanchi A, Davis JL, et al. Impact of Xpert MTB/RIF testing on tuberculosis management and outcomes in hospitalized patients in Uganda. PLoS One. 2012;7(11):e48599. https://doi.org/10.1371/journal.pone.0048599

16. Calligaro $\mathrm{GL}$, Theron $\mathrm{G}$, Khalfey $\mathrm{H}$, et al. Burden of tuberculosis in intensive care units in Cape Town, South Africa, and assessment of the accuracy and effect on patient outcomes of the Xpert MTB/RIF test on tracheal aspirate samples for diagnosis of pulmonary tuberculosis: A prospective burden of disease study with a nested randomised controlled trial. Lancet Respir Med. 2015;3(8):621-630. https://doi.org/10.1016/S2213-2600(15)00198-8

17. Steingart KR, Schiller I, Horne DJ, Pai M, Boehme CC, Dendukuri N. Xpert(R) MTB/RIF assay for pulmonary tuberculosis and rifampicin resistance in adults. Cochrane Database Syst Rev. 2014;(1):CD009593. https://doi.org/10.1002/14651858.CD009593.pub3

18. Kohli M, Schiller I, Dendukuri N, et al. Xpert. Cochrane Database Syst Rev. 2018;8:CD012768

19. Foundation for Innovative New Diagnostics, Geneva, Switzerland. Report: Performance of Xpert MTB/RIF Version G4 assay2011 November 2011. Available from: http://www.stoptb.org/wg/gli/assets/documents/map/findg4cartridge.pdf.

20. Osman M, Simpson JA, Caldwell J, Bosman M, Nicol MP. GeneXpert MTB/RIF version G4 for identification of rifampin-resistant tuberculosis in a programmatic setting. J Clin Microbiol. 2014;52(2):635-637. https://doi.org/10.1128/JCM 02517-13

21. Lawn SD, Kerkhoff $A D$, Burton $R$, et al. Rapid microbiological screening for tuberculosis in HIV-positive patients on the first day of acute hospital admission by systematic testing of urine samples using Xpert MTB/RIF: A prospective cohort in South Africa. BMC Med. 2015;13:192. https://doi.org/10.1186/s12916-015 $0432-2$

22. Gupta-Wright A, Corbett EL, Van Oosterhout JJ, et al. Rapid urine-based screening for tuberculosis in HIV-positive patients admitted to hospital in Africa (STAMP): A pragmatic, multicentre, parallel-group, double-blind, randomised controlled trial. Lancet. 2018;392(10144):292-301. https://doi.org/10.1016/ S0140-6736(18)31267-4

23. Lawn SD, Kerkhoff AD, Burton R, et al. Diagnostic accuracy, incremental yield and prognostic value of Determine TB-LAM for routine diagnostic testing for tuberculosis in HIV-infected patients requiring acute hospital admission in South Africa: A prospective cohort. BMC Med. 2017;15(1):67. https://doi.org/10.1186/ s12916-017-0822-8

24. Lawn SD, Brooks SV, Kranzer K, et al. Screening for HIV-associated tuberculosis and rifampicin resistance before antiretroviral therapy using the Xpert MTB/RIF assay: A prospective study. PLoS Med. 2011;8(7):e1001067. https://doi.org/10.1371/ journal.pmed.1001067

25. Lawn SD, Kerkhoff AD, Vogt M, Wood R. High diagnostic yield of tuberculosis from screening urine samples from HIV-infected patients with advanced immunodeficiency using the Xpert MTB/RIF assay. J Acquir Immune Defic Syndr. 2012;60(3):289-294. https://doi.org/10.1371/journal.pone.0054587

26. Pooran A, Pieterson E, Davids M, Theron G, Dheda K. What is the cost of diagnosis and management of drug resistant tuberculosis in South Africa? PLoS One. 2013;8(1):e54587. https://doi.org/10.1371/journal.pone.0054587

27. WHO meeting report of a technical expert consultation: Non-inferiority analysis of Xpert MTF/RIF Ultra compared to Xpert MTB/RIF. Geneva: World Health Organization; 2017.

28. Chakravorty S, Simmons AM, Rowneki M, et al. The new Xpert MTB/RIF ultra: Improving detection of mycobacterium tuberculosis and resistance to rifampin in an assay suitable for point-of-care testing. MBio. 2017;8(4):e00812-1. https://doi. org/10.1128/mBio.00812-17 
29. Dorman SE, Schumacher SG, Alland D, et al. Xpert MTB/RIF Ultra for detection of Mycobacterium tuberculosis and rifampicin resistance: A prospective multicentre diagnostic accuracy study. Lancet
org/10.1016/S1473-3099(17)30691-6

30. Kumar P, Balooni V, Sharma BK, Kapil V, Sachdeva KS, Singh S. High degree of multidrug resistance and hetero-resistance in pulmonary TB patients from Punjab state of India. Tuberculosis (Edinb). 2014;94(1):73-80. https://doi.org/10.1016/j. tube.2013.10.001

31. Kamakoli MK, Sadegh HR, Farmanfarmaei G, et al. Evaluation of the impact of polyclonal infection and heteroresistance on treatment of tuberculosis patients. Sci Rep. 2017;7:41410. https://doi.org/10.1038/srep41410
32. Cohen T, Van Helden PD, Wilson D, et al. Mixed-strain mycobacterium tuberculosis infections and the implications for tuberculosis treatment and control. Clin Microbiol Rev. 2012;25(4):708-719. https://doi.org/10.1128/CMR.00021-12

33. Van Deun A, Aung KJ, Bola V, et al. Rifampin drug resistance tests for tuberculosis: Challenging the gold standard. J Clin Microbiol. 2013;51(8):2633-2640. https:// doi.org/10.1128/JCM.00553-13

34. Rigouts L, Gumusboga M, De Rijk WB, et al. Rifampin resistance missed in automated liquid culture system for Mycobacterium tuberculosis isolates with specific rpoB mutations. J Clin Microbiol. 2013;51(8):2641-2645. https://doi. org/10.1128/JCM.02741-12 


\section{Appendix 1}

TABLE 1-A1: Patients with positive urine Xpert rifampicin resistant results - details of additional tuberculosis tests and clinical course.

\begin{tabular}{|c|c|c|c|c|c|c|c|c|c|c|}
\hline Study ID & $n$ & Sample type & Test & Organism & $\begin{array}{l}\text { Susceptibility } \\
\text { to rifampicin }\end{array}$ & $\begin{array}{l}\text { Susceptibility } \\
\text { to isoniazid }\end{array}$ & $\begin{array}{l}\text { 12-week } \\
\text { outcome }\end{array}$ & $\begin{array}{l}\text { Classification of } \\
\text { urine Xpert } \\
\text { rifampicin } \\
\text { resistance } \dagger\end{array}$ & Heteroresistance & Reason for classification \\
\hline \multirow[t]{2}{*}{ KDHTB067 } & 1 & Blood & Culture & MTB & - & - & \multirow[t]{2}{*}{ Died } & \multirow[t]{2}{*}{ Unknown } & \multirow[t]{2}{*}{ No } & \multirow{2}{*}{$\begin{array}{l}\text { No samples could confirm } \\
\text { resistance. Patient initially } \\
\text { improved on drug-sensitive TB } \\
\text { treatment and then experienced } \\
\text { rapid neurological deterioration } \\
\text { and died on day } 3 \text { of therapy. }\end{array}$} \\
\hline & 1 & Blood & Culture & Neg & - & - & & & & \\
\hline \multirow[t]{4}{*}{ KDHTB086 } & 2 & Blood & Culture & Neg & - & - & \multirow[t]{4}{*}{ Died } & \multirow[t]{4}{*}{ True Resistance } & \multirow[t]{4}{*}{ No } & \multirow{4}{*}{$\begin{array}{l}\text { Urine culture sample confirmed } \\
\text { rifampicin resistance and sputum } \\
\text { Xpert indicated rifampicin } \\
\text { resistance. }\end{array}$} \\
\hline & 1 & CSF & Culture & Neg & - & - & & & & \\
\hline & 1 & Sputm & Xpert & MTB & Resistant & - & & & & \\
\hline & 1 & Urine & Culture & MTB & Resistant & Resistant & & & & \\
\hline \multirow[t]{5}{*}{ KDHTB110 } & 1 & Blood & Culture & Neg & - & - & \multirow[t]{5}{*}{ Alive } & \multirow[t]{5}{*}{ True Resistance } & \multirow[t]{5}{*}{ No } & \multirow{5}{*}{$\begin{array}{l}\text { One blood and three urine culture } \\
\text { samples confirmed rifampicin } \\
\text { resistance. }\end{array}$} \\
\hline & 1 & Blood & Culture & МTB & Resistant & Resistant & & & & \\
\hline & 1 & CSF & Culture & Neg & - & - & & & & \\
\hline & 1 & Sputum & Culture & Neg & - & - & & & & \\
\hline & 3 & Urine & Culture & МTB & Resistant & Resistant & & & & \\
\hline \multirow[t]{5}{*}{ KDHTB203 } & 1 & Blood & Culture & MTB & - & - & \multirow[t]{5}{*}{ Alive } & \multirow[t]{5}{*}{ True Resistance } & Yes & Sputum culture result showed \\
\hline & 1 & Blood & Culture & MTB & Sensitive & Sensitive & & & & other cultures (sputum, blood \\
\hline & 1 & Sputum & Culture & MTB & Sensitive & - & & & & and urine) showed drug-sensitive \\
\hline & 1 & Sputum & Culture & MTB & Resistant & Sensitive & & & & \\
\hline & 1 & Urine & Culture & MTB & Sensitive & Sensitive & & & & \\
\hline KDHTB238 & 1 & Blood & Culture & МTB & Resistant & Sensitive & LTFU & True Resistance & No & Five cultures and one additional \\
\hline & 4 & Sputum & Culture & MTB & Resistant & Sensitive & & & & resistance. \\
\hline & 1 & Sputum & Xpert & MTB & Resistant & - & & & & \\
\hline KDHTB242 & 1 & Blood & Culture & Neg & - & - & Alive & True Resistance & No & Four cultures confirmed \\
\hline & 1 & Pleural & Culture & MTB & Resistant & Sensitive & & & & \\
\hline & 2 & Sputum & Culture & MTB & Resistant & Sensitive & & & & \\
\hline & 1 & Urine & Culture & MTB & Resistant & Sensitive & & & & \\
\hline KDHTB264 & 2 & Blood & Culture & Neg & - & - & Alive & True Resistance & No & One culture result confirmed \\
\hline & 1 & CSF & Culture & Neg & - & - & & & & \\
\hline & 1 & Pleural & Culture & MTB & Resistant & Resistant & & & & \\
\hline & 2 & Urine & Culture & $\mathrm{Neg}$ & - & - & & & & \\
\hline KDHTB398 & 2 & Blood & Culture & MTB & Resistant & Resistant & Died & True Resistance & No & Two culture results confirmed \\
\hline & 1 & CSF & Culture & Neg & - & - & & & & \\
\hline KDHTB416 & 1 & Blood & Culture & Neg & - & - & LTFU & True Resistance & No & Two culture results confirmed \\
\hline & 1 & Sputum & Culture & Neg & - & - & & & & \\
\hline & 2 & Sputum & Culture & MTB & Resistant & Resistant & & & & \\
\hline KDHTB439 & 1 & Blood & Culture & Neg & - & - & Alive & False Resistance & No & One culture and three Xpert \\
\hline & 1 & Pleural & Xpert & MTB & Sensitive & - & & & & $\begin{array}{l}\text { sensitivity. } \\
\text { siln }\end{array}$ \\
\hline & 1 & Sputum & Xpert & MTB & Sensitive & - & & & & \\
\hline & 1 & Sputum & Culture & MTB & Sensitive & Sensitive & & & & \\
\hline & 1 & Urine & Culture & Contam & - & - & & & & \\
\hline & 1 & Urine & Xpert & MTB & Sensitive & - & & & & \\
\hline KDHTB444 & 2 & Blood & Culture & МTB & - & - & Died & True Resistance & No & One culture result confirmed \\
\hline & 1 & Blood & Culture & MTB & Neg & - & & & & \\
\hline & 1 & Sputum & Culture & MTB & Resistant & Resistant & & & & \\
\hline KDHTB445 & 1 & Blood & Culture & Neg & - & - & Alive & True Resistance & No & Three culture results confirmed \\
\hline & 3 & Sputum & Culture & MTB & Resistant & Resistant & & & & \\
\hline KDHTB477 & 1 & Blood & Culture & МтВ & Resistant & Sensitive & Alive & True Resistance & No & Three culture results confirmed \\
\hline & 2 & Sputum & Culture & MTB & Resistant & Sensitive & & & & \\
\hline KDHTB479 & 1 & Blood & Culture & Neg & - & - & Alive & False Resistance & No & Two culture results showed \\
\hline & 2 & Sputum & Culture & MTB & Sensitive & Sensitive & & & & \\
\hline KDHTB524 & 1 & Blood & Culture & Neg & - & - & Alive & True Resistance & No & One culture and two Xpert results \\
\hline & 1 & Sputum & Culture & Neg & - & - & & & & \\
\hline & 2 & Sputum & Xpert & MTB & Resistant & - & & & & \\
\hline & 1 & Sputum & Culture & MTB & Resistant & Sensitive & & & & \\
\hline
\end{tabular}


TABLE 1-A1 (Continues...): Patients with positive urine Xpert rifampicin resistant results - details of additional tuberculosis tests and clinical course.

\begin{tabular}{|c|c|c|c|c|c|c|c|c|c|c|}
\hline Study ID & $n$ & Sample type & Test & Organism & $\begin{array}{l}\text { Susceptibility } \\
\text { to rifampicin }\end{array}$ & $\begin{array}{l}\text { Susceptibility } \\
\text { to isoniazid }\end{array}$ & $\begin{array}{l}\text { 12-week } \\
\text { outcome }\end{array}$ & $\begin{array}{l}\text { Classification } \\
\text { of urine Xpert } \\
\text { rifampicin } \\
\text { resistance } \dagger\end{array}$ & Heteroresistance & Reason for classification \\
\hline \multirow[t]{2}{*}{ KDHTB531 } & 1 & Blood & Culture & МTB & Sensitive & Sensitive & \multirow[t]{2}{*}{ Died } & \multirow[t]{2}{*}{ True Resistance } & \multirow[t]{2}{*}{ Yes } & \multirow{2}{*}{$\begin{array}{l}\text { One culture result confirmed } \\
\text { rifampicin resistance and one } \\
\text { showed rifampicin sensitivity. }\end{array}$} \\
\hline & 1 & Sputum & Culture & MTB & Resistant & Sensitive & & & & \\
\hline \multirow[t]{3}{*}{ KDHTB555 } & 1 & Blood & Culture & MTB & Resistant & Resistant & \multirow[t]{3}{*}{ Alive } & \multirow[t]{3}{*}{ True Resistance } & \multirow[t]{3}{*}{ No } & \multirow{3}{*}{$\begin{array}{l}\text { Three culture results confirmed } \\
\text { rifampicin resistance. }\end{array}$} \\
\hline & 2 & Blood & Culture & Neg & - & - & & & & \\
\hline & 2 & Sputum & Culture & МTB & Resistant & Resistant & & & & \\
\hline \multirow[t]{3}{*}{ KDHTB556 } & 1 & Abscess & Culture & MTB & Resistant & Resistant & \multirow[t]{3}{*}{ Died } & \multirow[t]{3}{*}{ True Resistance } & \multirow[t]{3}{*}{ No } & \multirow{3}{*}{$\begin{array}{l}\text { Two culture results confirmed } \\
\text { rifampicin resistance. }\end{array}$} \\
\hline & 1 & Blood & Culture & Neg & - & - & & & & \\
\hline & 1 & Sputum & Culture & MTB & Resistant & Resistant & & & & \\
\hline \multirow[t]{2}{*}{ KDHTB560 } & 2 & Blood & Culture & Neg & - & - & \multirow[t]{2}{*}{ Alive } & \multirow[t]{2}{*}{ True Resistance } & \multirow[t]{2}{*}{ No } & One culture result confirmed \\
\hline & 1 & Sputum & Culture & MTB & Resistant & Resistant & & & & \\
\hline KDHTB598 & 1 & Blood & Culture & MTB & Resistant & Sensitive & Died & True Resistance & No & Three culture results confirmed \\
\hline & 1 & CSF & Culture & MTB & Resistant & Sensitive & & & & \\
\hline & 1 & Sputum & Culture & МTB & Resistant & Sensitive & & & & \\
\hline KDHTB616 & 1 & Abscess & Culture & MTB & Resistant & Sensitive & Alive & True Resistance & No & Four culture results confirmed \\
\hline & 2 & Blood & Culture & MTB & Resistant & Sensitive & & & & \\
\hline & 5 & Blood & Culture & Neg & - & - & & & & \\
\hline & 1 & Blood & Culture & МTB & Resistant & Sensitive & & & & \\
\hline KDHTB627 & 1 & Blood & Culture & MTB & Resistant & Sensitive & Died & True Resistance & No & $\begin{array}{l}\text { One culture result confirmed } \\
\text { rifampicin resistance. }\end{array}$ \\
\hline KDHTB631 & 1 & Blood & Culture & Neg & - & - & Alive & True Resistance & No & One Xpert result and two culture \\
\hline & 2 & Sputum & Culture & MTB & Resistant & Resistant & & & & resistance. \\
\hline & 1 & Sputum & Xpert & MTB & Resistant & - & & & & \\
\hline & 1 & Urine & Culture & Neg & - & - & & & & \\
\hline KDHTB660 & 6 & Blood & Culture & Neg & - & - & Died & True Resistance & No & Two culture results confirmed \\
\hline & 2 & Blood & Culture & МTB & Resistant & Sensitive & & & & \\
\hline KDHTB668 & 1 & Blood & Culture & Neg & - & - & Alive & True Resistance & No & $\begin{array}{l}\text { Treated for MDR-TB, improved } \\
\text { and survived. }\end{array}$ \\
\hline JTBS021 & 1 & Blood & Culture & Neg & - & - & Alive & Unknown & No & No samples confirmed rifampicin \\
\hline & 1 & Pleural eff & Culture & Neg & - & - & & & & $\begin{array}{l}\text { drug-sensitive TB treatment. } \\
\text { Inadequate information on } \\
\text { improvement or deterioration. }\end{array}$ \\
\hline JTBS074 & 1 & Urine & Xpert & Neg & - & - & Alive & False Resistance & No & $\begin{array}{l}\text { No sample confirmed resistance } \\
\text { and patient improved on }\end{array}$ \\
\hline & 1 & Blood & Culture & Neg & - & - & & & & drug-sensitive TB treatment. \\
\hline & 1 & CSF & Culture & Neg & - & - & & & & \\
\hline JTBS090 & 2 & Urine & Culture & MTB & Resistant & Resistant & LTFU & True Resistance & No & Three culture results confirmed \\
\hline & 1 & Sputum & Culture & MTB & Resistant & Resistant & & & & \\
\hline JTBS097† & 2 & Blood & Culture & MTB & Sensitive & Sensitive & Died & Contamination & No & Two culture results showed \\
\hline & 1 & Sputum & Culture & Neg & - & - & & & & started drug-sensitive TB \\
\hline & 1 & Sputum & Culture & MTB & Sensitive & Sensitive & & & & treatment, then switched to \\
\hline & 1 & Urine & Culture & Neg & - & - & & & & urine Xpert result; however, died \\
\hline & 1 & Sputum & Culture & Contam & - & - & & & & $\begin{array}{l}\text { before other drug susceptibility } \\
\text { results were available }\end{array}$ \\
\hline & 1 & CSF & Culture & Contam & - & - & & & & \\
\hline JTBS098 & 1 & Urine & Xpert & Neg & - & - & Died & True Resistance & No & No samples from enrolment \\
\hline & 1 & Blood & Culture & Neg & - & - & & & & $\begin{array}{l}\text { admission connmed ritampicin } \\
\text { resistance; however, a 7-month- }\end{array}$ \\
\hline & 2 & CSF & Culture & Neg & - & - & & & & old result showed rifampicin \\
\hline & 1 & Sputum & Culture & Contam & - & - & & & & $\begin{array}{l}\text { was untreated. It is unclear why } \\
\text { the patient was not treated for } \\
\text { MDR-TB and he died shortly after } \\
\text { admission to hospital. }\end{array}$ \\
\hline JTBS099† & 4 & $\begin{array}{l}\text { Tracheal } \\
\text { aspirate }\end{array}$ & Culture & MTB & Sensitive & Resistant & Alive & Contamination & No & $\begin{array}{l}\text { Four culture results showed } \\
\text { rifampicin sensitivity and patient } \\
\text { responded to drug-sensitive TB } \\
\text { treatment. }\end{array}$ \\
\hline JTBS159 & 1 & Urine & Xpert & Neg & - & - & Alive & False Resistance & No & No cultures confirmed rifampicin \\
\hline & 1 & Blood & Culture & Neg & - & - & & & & $\begin{array}{l}\text { resistance and one sputum Xpert } \\
\text { showed rifampicin sensitivity. }\end{array}$ \\
\hline & 2 & Sputum & Culture & Neg & - & - & & & & Patient treated with drug- \\
\hline & 1 & Sputum & Xpert & MTB & Sensitive & - & & & & improved. \\
\hline & 1 & Sputum & Culture & Contam & - & - & & & & \\
\hline & 1 & Urine & Culture & Neg & - & - & & & & \\
\hline
\end{tabular}


TABLE 1-A1 (Continues...): Patients with positive urine Xpert rifampicin resistant results - details of additional tuberculosis tests and clinical course.

\begin{tabular}{|c|c|c|c|c|c|c|c|c|c|c|}
\hline Study ID & $n$ & Sample type & Test & Organism & $\begin{array}{l}\text { Susceptibility } \\
\text { to rifampicin }\end{array}$ & $\begin{array}{l}\text { Susceptibility } \\
\text { to isoniazid }\end{array}$ & $\begin{array}{l}\text { 12-week } \\
\text { outcome }\end{array}$ & $\begin{array}{l}\text { Classification } \\
\text { of urine Xpert } \\
\text { rifampicin } \\
\text { resistance } \dagger\end{array}$ & Heteroresistance & Reason for classification \\
\hline \multirow[t]{3}{*}{ JTBS160 } & 1 & Blood & Culture & Neg & - & - & \multirow[t]{3}{*}{ Alive } & \multirow{3}{*}{$\begin{array}{l}\text { False Resistance } \\
\text { (on two results) }\end{array}$} & \multirow[t]{3}{*}{ No } & \multirow{3}{*}{$\begin{array}{l}\text { Two cultures showed rifampicin } \\
\text { sensitivity and patient improved } \\
\text { on drug-sensitive TB treatment. }\end{array}$} \\
\hline & 2 & Sputum & Culture & MTB & Sensitive & Sensitive & & & & \\
\hline & 1 & Urine & Culture & Neg & - & - & & & & \\
\hline \multirow[t]{4}{*}{ JTBS181 } & 1 & Urine & Xpert & MTB & Resistant & - & \multirow[t]{4}{*}{ Alive } & \multirow{4}{*}{$\begin{array}{l}\text { True Resistance } \\
\text { (on two results) }\end{array}$} & \multirow[t]{4}{*}{ No } & \multirow{4}{*}{$\begin{array}{l}\text { Two culture results confirmed } \\
\text { rifampicin resistance. }\end{array}$} \\
\hline & 1 & Sputum & Culture & MTB & Resistant & - & & & & \\
\hline & 2 & Ascites & Culture & Neg & - & - & & & & \\
\hline & 1 & Urine & Culture & MTB & Resistant & Resistant & & & & \\
\hline \multirow[t]{5}{*}{ JTBS192 } & 1 & Sputum & Culture & MTB & Resistant & - & \multirow[t]{5}{*}{ Died } & \multirow[t]{5}{*}{ True Resistance } & \multirow[t]{5}{*}{ No } & \multirow{5}{*}{$\begin{array}{l}\text { Three culture results confirmed } \\
\text { rifampicin resistance. }\end{array}$} \\
\hline & 1 & Urine & Xpert & Neg & - & - & & & & \\
\hline & 1 & Urine & Culture & MTB & Resistant & Resistant & & & & \\
\hline & 1 & Sputum & Culture & MTB & Resistant & Resistant & & & & \\
\hline & 1 & Sputum & Culture & Neg & - & - & & & & \\
\hline \multirow[t]{4}{*}{ JTBS202 } & 1 & Urine & Xpert & Neg & - & - & \multirow[t]{4}{*}{ Alive } & \multirow[t]{4}{*}{ False Resistance } & \multirow[t]{4}{*}{ No } & \multirow{4}{*}{$\begin{array}{l}\text { One culture result showed } \\
\text { rifampicin sensitivity and patient } \\
\text { improved on drug-sensitive TB } \\
\text { treatment }\end{array}$} \\
\hline & 1 & Blood & Culture & Neg & - & - & & & & \\
\hline & 1 & Sputum & Culture & Neg & - & - & & & & \\
\hline & 1 & Sputum & Culture & MTB & Sensitive & Sensitive & & & & \\
\hline \multirow[t]{2}{*}{ JTBS211 } & 9 & Sputum & Culture & Neg & - & - & \multirow[t]{2}{*}{ Alive } & \multirow[t]{2}{*}{ False Resistance } & \multirow[t]{2}{*}{ No } & No confirmation of rifampicin \\
\hline & 1 & Sputum & Culture & Contam & - & - & & & & $\begin{array}{l}\text { resistance patient Improved on } \\
\text { drug-sensitive TB treatment. }\end{array}$ \\
\hline JTBS249 & 1 & Urine & Xpert & Neg & - & - & Alive & False Resistance & No & No confirmation of rifampicin \\
\hline & 1 & Blood & Xpert & Neg & - & - & & & & on drug-sensitive TB treatment. \\
\hline & 5 & CSF & Culture & Neg & & & & & & \\
\hline JTBS358 & 1 & Urine & Xpert & MTB & Sensitive & - & Alive & False Resistance & No & No cultures confirmed rifampicin \\
\hline & 1 & Urine & Xpert & Neg & - & - & & & & on drug-sensitive TB treatment. \\
\hline & 1 & Sputum & Culture & MTB & - & - & & & & \\
\hline & 1 & Blood & Culture & Neg & - & - & & & & \\
\hline JTBS534 & 1 & Urine & Xpert & Neg & - & - & Died & True Resistance & No & No samples confirmed rifampicin \\
\hline & 1 & Blood & Culture & Neg & - & - & & & & $\begin{array}{l}\text { resistance. Patient presented with } \\
\text { ascites and pleural fluid of which }\end{array}$ \\
\hline & 1 & Ascites & Culture & Neg & - & - & & & & all TB tests were negative. Ascitic \\
\hline & 1 & Pleural & Culture & Neg & - & - & & & & $\begin{array}{l}\text { fluid had high ADA and patient } \\
\text { was started on drug-sensitive TB }\end{array}$ \\
\hline & 1 & Sputum & Culture & Neg & - & - & & & & $\begin{array}{l}\text { treatment. About } 4 \text { weeks later, } \\
\text { the patient was readmitted while } \\
\text { clinically deteriorating on } \\
\text { drug-sensitive TB treatment. A } \\
\text { urine Xpert result showed } \\
\text { rifampicin resistance; however, } \\
\text { the patient died shortly after } \\
\text { receipt of urine Xpert result. }\end{array}$ \\
\hline JTBS574 & 1 & Urine & Xpert & Neg & - & - & Alive & False Resistance & No & One culture and two Xpert results \\
\hline & 1 & Blood & Culture & Neg & - & - & & & & $\begin{array}{l}\text { showed rifampicin sensitivity and } \\
\text { patient improved on drug- }\end{array}$ \\
\hline & 1 & Sputum & Culture & MTB & Sensitive & Sensitive & & & & sensitive TB treatment. \\
\hline & 2 & Sputum & Xpert & MTB & Sensitive & - & & & & \\
\hline
\end{tabular}

$n=$ number of samples; - Susceptibility unknown or not available. Neg, negative; Contam, Contaminated; MTB, Mycobacterial tuberculosis; LTFU, lost to follow-up; TB, tuberculosis; Pleural, Pleura effusion; Abscess, Abscess aspirate; Drug-sensitive TB treatment = Intensive phase with isoniazid, rifampicin, ethambutol and pyrazinamide, followed by consolidation phase with isoniazid and rifampicin.

$\dagger$, Two patients had false urine Xpert rifampicin resistance due to contamination (accounting for three results). These patients were excluded from analysis.

For JTBS patients, nearly all had their urine specimens tested by Xpert using two different methods (unconcentrated and concentrated). This accounts for why patients may have two urine Xpert results. However, in the table above, rifampicin resistance was only detected on one urine Xpert result, unless stated otherwise. Some patients may have had an additional urine Xpert performed for confirmation after receiving a rifampicin resistant result.

Of note, the table includes all other TB cultures and Xperts conducted on all clinical specimens, including repeat urine Xpert tests conducted. 